\title{
Effects of Task-Irrelevant Arousal on State Transition Learning
}

\author{
Paul Sharp ${ }^{1,2}$, Raymond Dolan ${ }^{1,2}$, and Eran Eldar ${ }^{3,1}$
}

1 Max Planck UCL Centre for Computational Psychiatry and Ageing Research, 2 University College London; Wellcome Centre for Human Neuroimaging, 3 The Hebrew University of Jerusalem

Background: Anxiety is characterized by sympathetic hyperarousal and beliefs that aversive events are more likely than is truly the case. Here we combine computational insights into how individuals learn about the structure of their environment with an arousal manipulation to ask how the latter might bias learning of probabilistic task transitions.

Methods: We conducted two on-line studies $(n=135, n=174)$ to test how rapid presentations of task-irrelevant emotional stimuli, interposed between state transitions, influenced individuals estimates of those transitions. Using a one-step revaluation task, participants were told to ignore the brief emotional stimuli and learn the most common task transition an action led to.

Results: In study 1, we found evidence that when actions led to two outcomes with equal probability, individuals learned more from transitions paired with personally-salient negative words than those paired with neutral words $(\mathrm{t}(135))=-4.37, \mathrm{p}=.0002)$. Study 2 tested if taskirrelevant images, that were rated high in arousal, biased learning in a more difficult version of the same task. A Bayesian model provided support for both positive and negative arousal exerting a differential impact on learning: negative arousal improved learning for states that succeeded it, whereas positive arousal compromised this effect.

Conclusions: Preliminary evidence supports that task-irrelevant emotional stimuli impact modelbased learning about the structure of one's environment. Moreover, negative arousal enhanced learning whereas positive arousal diminished learning. The findings have implications for theories of aberrant learning in psychopathology.

Supported By: Max Planck UCL Centre for Computational Psychiatry and Ageing Research Keywords: Anxiety, Computational Modeling, Reinforcement Learning 